\title{
Research on Wear State of Vehicles Engine Based on Matter-Element Method
}

\author{
Tang Yanfeng ${ }^{1, a}$, Zhang Jian ${ }^{1, b}$, Liu Xiangkai ${ }^{1, c}$ \\ ${ }^{1}$ Automobile Engineering Department, Military Transportation University, Tianjin 300161,China \\ ajjxy_tang@163.com, byitzhak@163.com, cliu_xkai@126.com
}

Keywords: Vehicles Engine; Wear State Monitoring; Oil Spectrum Analysis; Matter-Element

\begin{abstract}
An evaluation method based on matter-element analysis theory was purposed in this paper, because of the method to evaluate the engine wear state monitoring based on oil spectrum analysis presently had the shortage such as too many human factors and uncertainty weight calculations. According to the various indicators of the comprehensive evaluation during engine wear state monitoring and the associate degree calculated, the precise monitoring of engine wear state can be realized. The example has showed that the method purposed in this paper is scientific and reliable, and it had further researching and applying value in the area of engine wear state monitoring based on oil spectrum analysis
\end{abstract}

\section{Introduction}

Engine wear is determined by many factors and it is a complex process to take the comprehensive evaluation for engine wear state. Fuzzy mathematical method is adopted presently ${ }^{[1]}$. The key of the fuzzy evaluation method is the membership degree of artificial selection by researchers. Even if evaluating the same research objects, different researchers may select the different membership degree, along with the widely different evaluation results. There also exist the shortages such as extremum covered-up, information lost, maximum membership principle and so on in the comprehensive evaluation process ${ }^{[2]}$.

There are two pillars to support the theory framework of matter-element analysis ${ }^{[3]}$. One is the matter-element theory researching the matter-element and its changes, and the other is the mathematical tools based on the extension set. In the matter-element analysis, triples of things, characteristics and magnitude are the basic element to describe things, which can be recorded as $R=$ ( $P, C, Y$ ), where $P$ refers to things, $C$ refers to the characteristics of things, and $Y$ is the magnitude of C.

It can research the matter-element's extensibility by extension set in matter-element analysis, and the extension set is portrayed by the associate function and the specific restrictions of matter-element, and thus the basic framework of matter-element analysis is constituted. Applying the matter-element analysis theory to the engine wear state monitoring process based on oil spectrum analysis, through the establishment of the evaluation model of multiple performance parameters, the evaluation results can be accurately represented by quantitative values applying the correlation function in matter-element analysis to evaluate. Thereby, it is possible to evaluate the wear state of the engine comprehensively.

\section{Construction of the Matter-Element Model}

Matter-element is the basic element of the things, including the characteristics and the money of things. If things is described by $n$ characteristics as $c_{1}, c_{2}, \ldots, c_{n}$ and their corresponding money as $v_{1}$, $v_{2}, \ldots, v_{n}$, then $R$ can be expressed as: $R=\left[\begin{array}{cc}c_{1} & v_{1} \\ c_{2} & v_{2} \\ \vdots & \vdots \\ c_{n} & v_{n}\end{array}\right]$

After the introduction of the concept of matter-element, the oil spectroscopy data can be 
represented by matter element, and thus a comprehensive evaluation can be make for wear state. The steps are as follows.

\section{Determine the classical domain}

Since there are many friction pairs in an engine, the frication pairs that fully contains wear information in lubricant should focus be considered, such as the cylinder liner and piston, cylinder liner and gas ring, piston and piston pin, bearings shell and crankshaft, camshaft and cam bearings, inclined shaft gear and camshaft gear, and so on. Selecting the wear characteristics effectively is the key to evaluate the wear state of engines According to material composition of the engines' friction pair and its sensitivity to the wear state, select four elements as $\mathrm{Fe}, \mathrm{Cu}, \mathrm{Al}, \mathrm{Cr}$ to be the wear characterize elements, which concentration values as C1, C2, C3, C4 and gradient values as C5, C6, C7, C8 can be obtained through spectral analysis ${ }^{[4]}$. They can conduct the evidence to evaluate engine wear state.

From the point of engineering applications, engine wear state can be divided into three assessment levels of wear state as normal wear state, warning wear state and abnormal wear state, which can be respectively indicated by 1,2 and 3 . That is, engines run normally when it is in the normal wear state, engines can run normally but must take the operators' attention to carry out level maintenance appropriately when it is in the warning wear state, and engines should stop using and start disassembling and maintenance when it is in the warning wear state. Therefore, the oil spectroscopy data in each wear state can be represented as the following matter-element model ${ }^{[5]}$.

$$
R^{j}=\left[\begin{array}{cc}
c_{1} & X_{1}^{j} \\
c_{2} & X_{2}^{j} \\
\vdots & \vdots \\
c_{8} & X_{8}^{j}
\end{array}\right]=\left[\begin{array}{cc}
c_{1} & \left\langle a_{1}^{j}, b_{1}^{j}\right\rangle \\
c_{2} & \left\langle a_{2}^{j}, b_{2}^{j}\right\rangle \\
\vdots & \vdots \\
c_{8} & \left\langle a_{8}^{j}, b_{8}^{j}\right\rangle
\end{array}\right]
$$

Wherein, $j=1$, 2, 3; $R^{j}$ represents the oil spectral matter-element in the $j$-th level; $X_{k}^{j}=\left\langle a_{k}^{j}, b_{k}^{j}\right\rangle(k=$ $1,2, \ldots, 8)$ represents the magnitude range of the $k$-th evaluation indicators when in the $j$-th wear state. Classical domain $R^{j}(j=1,2,3)$ is the magnitude range of all the evaluation indicators of the engine oil which correspond with the each wear state.

\section{Determine node region}

Determine the allowable range of the engine oil's evaluation indicators through data analysis, expressed as follows matter-element.

$$
R^{p}=\left[\begin{array}{cc}
c_{1} & X_{1}^{p} \\
c_{2} & X_{2}^{p} \\
\vdots & \vdots \\
c_{8} & X_{8}^{p}
\end{array}\right]=\left[\begin{array}{cc}
c_{1} & \left\langle a_{1}^{p}, b_{1}^{p}\right\rangle \\
c_{2} & \left\langle a_{2}^{p}, b_{2}^{p}\right\rangle \\
\vdots & \vdots \\
c_{8} & \left\langle a_{8}^{p}, b_{8}^{p}\right\rangle
\end{array}\right]
$$

Wherein, $R^{p}$ represents the matter-elements of oil spectrum analysis in each wear state; $X_{k}^{p}=\left\langle a_{k}^{p}, b_{k}^{p}\right\rangle(k=1,2 \ldots 8)$ represents the allowable range of the $\mathrm{k}$-th evaluation indicator of engine oil. Node region $R^{p}$ is the allowable range of all evaluation indicators of engine oil, with $X_{k}^{j} \subset X_{k}^{p}(j=1,2,3 ; k=1,2, \cdots, 8)$ apparently. For the sake of brevity, all the $X_{k}^{j}=\left\langle a_{k}^{j}, b_{k}^{j}\right\rangle$ in classical domain and all the $X_{k}^{p}=\left\langle a_{k}^{p}, b_{k}^{p}\right\rangle$ in node region can be unified to the same list, as Table 2 and Table 3.

\section{Determine the spectral analysis matter-element}

Collate the various measurement results of the engine lubricating oil to be evaluated, and represented as the following matter-element. $R=\left[\begin{array}{cc}c_{1} & v_{1} \\ c_{2} & v_{2} \\ \vdots & \vdots \\ c_{n} & v_{n}\end{array}\right]$

Wherein, $R$ represents the oil spectrum analysis matter-element to be evaluated, $v_{k} \quad(k=1,2,3 \ldots . .8)$ represents the evaluation value about the oil's $k$-th indicator. 


\section{Evaluation Method for Wear Monitoring Oil Spectrum’s Matter-Element Model}

According to the matter-element model of the oil spectrum analysis in wear monitoring that has been established, in order to evaluate the level of the engine's wear, it is necessary to calculate the associate degree among all wear levels of the engine's lubricating oil to be evaluated. Then the wear level of the engine to be evaluated can be determined according to the associate degree.

Calculate the associate degree for each evaluation value of engine's lubricating oil to be $\operatorname{evaluated}(R)$

Taken $j=1,2,3$ and $k=1,2, \ldots 8 . \mathrm{v}_{\mathrm{k}}$ is the evaluation value of the engine's lubricating oil to be evaluated about the $k$-th evaluation value, and $k_{j}\left(v_{k}\right)$ is the associate degree about the $j$-th wear level.

That is, the approach degree between $v_{k}$ and interval $X_{k}^{j}, X_{k}^{p}$ is

$$
k_{j}\left(v_{k}\right)=\left\{\begin{array}{c}
\frac{\rho\left(v_{k}, X_{k}^{j}\right)}{\rho\left(v_{k}, X_{k}^{p}\right)-\rho\left(v_{k}, X_{k}^{j}\right)}, \rho\left(v_{k}, X_{k}^{p}\right)-\rho\left(v_{k}, X_{k}^{j}\right) \neq 0 \\
-\rho\left(v_{k}, X_{k}^{j}\right)-1, \rho\left(v_{k}, X_{k}^{p}\right)-\rho\left(v_{k}, X_{k}^{j}\right)=0
\end{array}\right.
$$

in which

$$
\begin{aligned}
& \rho\left(v_{k}, X_{K}^{P}\right)=\left|v_{k}-\frac{b_{k}^{p}+a_{k}^{p}}{2}\right|-\frac{b_{k}^{p}-a_{k}^{p}}{2} \\
& \rho\left(v_{k}, X_{K}^{j}\right)=\left|v_{k}-\frac{b_{k}^{j}+a_{k}^{j}}{2}\right|-\frac{b_{k}^{j}-a_{k}^{j}}{2}
\end{aligned}
$$

\section{Determine the weights of the associate degree for each evaluation value of $\boldsymbol{R}$}

$$
W_{k}=\frac{v_{k}}{\bar{\psi}_{k}} / \sum_{i=1}^{8} \frac{v_{i}}{\bar{\psi}_{k}} \quad(\mathrm{k}=1,2, \ldots, 8)
$$

Wherein, $\bar{\psi}_{k}$ is the standard average of every wear level about the $k$-th evaluation indicator for lubricating oil.

Calculate the associate degree of $\boldsymbol{R}$

$$
K_{j}(R)=\sum_{k=1}^{8} W_{k} K_{j}\left(v_{k}\right) \quad(\mathbf{j}=1,2,3)
$$

\section{Normalize the associate degree of $R$}

$$
\bar{K}_{j}(R)=\left[K_{j}(R)-\min _{j=1}^{3} K_{j}(R)\right] /\left[\max _{j=1}^{3} K_{j}(R)-\min _{j=1}^{3} K_{j}(R)\right] \quad(\mathrm{j}=1,2,3)
$$

\section{Determine the wear level of engine to be evaluated}

If $\bar{K}_{j}(R)=\max _{j=1}^{3} K_{j}(R)$, the wear level of engine to be evaluated is $J$.

Set $\bar{J}=\sum_{j=1}^{3} j \bar{K}_{j}(R) / \sum_{j=1}^{3} \bar{K}_{j}(R)$

Then it can be seen from $\bar{J}$ that extent $R$ trends to the adjacent wear levels. For example, $\bar{J}=$ 1.6415 , then the wear state of engine is can be seen to trend to the second wear level which is warning. It can be obtained that the accurate wear level of engine to be evaluated is 1.6415.

\section{Examples}

\section{Data of Spectral analysis for oil samples}

The engine provided by a Military Division Training Battalion is continuously sampled, and two of the oil samples have been extracted. Analyzed by the SPECTROIL $M$ spectrometer, the concentration values of the major wear characteristics element have been obtained, as shown in Table 1.

Table1 concentration values of the major wear characteristics element 


\begin{tabular}{|c|c|c|c|c|}
\hline & $\mathrm{Fe}$ & $\mathrm{Cu}$ & $\mathrm{Al}$ & $\mathrm{Cr}$ \\
\hline 26400 & 21.5 & 4.9 & 7.6 & 2.8 \\
\hline 31400 & 37.5 & 10.6 & 7.6 & 4.7 \\
\hline
\end{tabular}

\section{The interval of evaluation indicators}

According to the expert experience and the statistical results, contrary to the wear state at all levels of the engine for a certain vehicle, the entire range of the concentration value and gradient value of the wear characterization element has been divided into three intervals of the normal area, the warning area and the abnormal area. Take the mean value of the engine's normal samples data as normal value, take the normal value plus two times variance of the samples as the warning value, and take the normal value plus three times variance of the samples as the abnormal values. Then the classical domain and the node region for the wear characterization elements calculated above are as shown in Table 2 and Table 3.

Table 2 the value interval of evaluation indicator in $R^{j}$

\begin{tabular}{|c|c|c|c|}
\hline \multirow{2}{*}{$\mathrm{k}$} & \multicolumn{3}{|c|}{$\mathrm{j}$} \\
\cline { 2 - 4 } & 1 & 2 & 3 \\
\hline 1 & $(0,90)$ & $(90,120)$ & $(120,500)$ \\
\hline 2 & $(0,10)$ & $(10,16)$ & $(16,40)$ \\
\hline 3 & $(0,20)$ & $(20,25)$ & $(30,78)$ \\
\hline 4 & $(0,17)$ & $(17,30)$ & $(8.8 \times 10-3,12.6 \times 10-3)$ \\
\hline 5 & $(0,5.4 \times 10-3)$ & $(5.4 \times 10-3,8.8 \times 10-3)$ & $(1.6 \times 10-3,4.8 \times 10-3)$ \\
\hline 6 & $(0,0.6 \times 10-3)$ & $(0.6 \times 10-3,1.6 \times 10-3)$ & $(2.2 \times 10-3,7.4 \times 10-3)$ \\
\hline 7 & $(0,1.0 \times 10-3)$ & $(1.0 \times 10-3,2.2 \times 10-3)$ & $(2.4 \times 10-3,4.2 \times 10-3)$ \\
\hline 8 & $(0,1.2 \times 10-3)$ & $(1.2 \times 10-3,2.4 \times 10-3)$ & \\
\hline
\end{tabular}

Table 3 the value interval of evaluation indicator in $R^{p}$

\begin{tabular}{|c|c|c|c|c|}
\hline $\mathrm{k}$ & 1 & 2 & 3 & 4 \\
\hline$\left\langle a_{k}^{p}, b_{k}^{p}\right\rangle$ & $(0,500)$ & $(0,40)$ & $(0,55)$ & $(0,78)$ \\
\hline $\mathrm{k}$ & & & & \\
\hline$\left\langle a_{k}^{p}, b_{k}^{p}\right\rangle$ & 5 & 6 & 7 & 8 \\
\hline
\end{tabular}

\section{Calculating and determine the wear levels}

(1) Constructing the oil spectrum matter-element R and calculate the corresponding evaluation value. The evaluation value about eight indicators of the certain vehicle engine oil's two sampling can be calculated by the data in Table 1, as shown in Table 4.

Table 4 evaluation value about eight indicators

\begin{tabular}{|c|c|c|c|c|c|c|c|}
\hline $\mathrm{V}_{1}$ & $\mathrm{~V}_{2}$ & $\mathrm{~V}_{3}$ & $\mathrm{~V}_{4}$ & $\mathrm{~V}_{5}$ & $\mathrm{~V}_{6}$ & $\mathrm{~V}_{7}$ & $\mathrm{~V}_{8}$ \\
\hline 21.5 & 4.9 & 7.6 & 2.8 & $3.22 \times 10^{-3}$ & $1.14 \times 10^{-3}$ & $0.18 \times 10^{-3}$ & $0.38 \times 10^{-3}$ \\
\hline
\end{tabular}

(2) Calculate the association degree of every indicator's evaluation value of the engine lubricants $\mathrm{R}$, as shown in Table 5 .

Table 5 association degree of every indicator's evaluation value of $R$

\begin{tabular}{|c|c|c|c|c|c|}
\hline $\mathrm{K}_{1}\left(\mathrm{~V}_{1}\right)$ & $\mathrm{K}_{2}\left(\mathrm{~V}_{1}\right)$ & $\mathrm{K}_{3}\left(\mathrm{~V}_{1}\right)$ & $\mathrm{K}_{1}\left(\mathrm{~V}_{2}\right)$ & $\mathrm{K}_{2}\left(\mathrm{~V}_{2}\right)$ & $\mathrm{K}_{3}\left(\mathrm{~V}_{2}\right)$ \\
\hline 20.5 & -0.761 & -0.821 & 3.9 & -0.51 & -0.65 \\
\hline \hline $\mathrm{K}_{1}\left(\mathrm{~V}_{3}\right)$ & $\mathrm{K}_{2}\left(\mathrm{~V}_{3}\right)$ & $\mathrm{K}_{3}\left(\mathrm{~V}_{3}\right)$ & $\mathrm{K}_{1}\left(\mathrm{~V}_{4}\right)$ & $\mathrm{K}_{2}\left(\mathrm{~V}_{4}\right)$ & $\mathrm{K}_{3}\left(\mathrm{~V}_{4}\right)$ \\
\hline 6.6 & -0.62 & -0.696 & 1.8 & -0.835 & -0.907 \\
\hline \hline $\mathrm{K}_{1}\left(\mathrm{~V}_{5}\right)$ & $\mathrm{K}_{2}\left(\mathrm{~V}_{5}\right)$ & $\mathrm{K}_{3}\left(\mathrm{~V}_{5}\right)$ & $\mathrm{K}_{1}\left(\mathrm{~V}_{6}\right)$ & $\mathrm{K}_{2}\left(\mathrm{~V}_{6}\right)$ & $\mathrm{K}_{3}\left(\mathrm{~V}_{6}\right)$ \\
\hline 2.096 & -0.404 & -0.634 & -0.321 & 0.676 & -0.288 \\
\hline \hline $\mathrm{K}_{1}\left(\mathrm{~V}_{7}\right)$ & $\mathrm{K}_{2}\left(\mathrm{~V}_{7}\right)$ & $\mathrm{K}_{3}\left(\mathrm{~V}_{7}\right)$ & $\mathrm{K}_{1}\left(\mathrm{~V}_{8}\right)$ & $\mathrm{K}_{2}\left(\mathrm{~V}_{8}\right)$ & $\mathrm{K}_{3}\left(\mathrm{~V}_{8}\right)$ \\
\hline-0.82 & -0.82 & -0.918 & -0.62 & -0.068 & -0.842 \\
\hline
\end{tabular}

(3) Calculate weights of association degree for each indicator's evaluation value, as shown in Table 6.

Table 6 the weights of association degree for every indicator's evaluation value 


\begin{tabular}{|c|c|c|c|c|c|c|c|}
\hline $\bar{\psi}_{1}$ & $\bar{\psi}_{2}$ & $\bar{\psi}_{3}$ & $\bar{\psi}_{4}$ & $\bar{\psi}_{5}$ & $\bar{\psi}_{6}$ & $\bar{\psi}_{7}$ & $\bar{\psi}_{8}$ \\
\hline 166.667 & 13.333 & 18.333 & 26.000 & $4.2 \times 10^{-3}$ & $1.6 \times 10^{-3}$ & $2.5 \times 10^{-3}$ & $1.4 \times 10^{-3}$ \\
\hline $\mathrm{W}_{1}$ & $\mathrm{~W}_{2}$ & $\mathrm{~W}_{3}$ & $\mathrm{~W}_{4}$ & $\mathrm{~W}_{5}$ & $\mathrm{~W}_{6}$ & $\mathrm{~W}_{7}$ & $\mathrm{~W}_{8}$ \\
\hline 0.045 & 0.129 & 0.146 & 0.038 & 0.27 & 0.251 & 0.026 & 0.095 \\
\hline
\end{tabular}

(4) Calculate the association degree for engine lubricants $R$, as shown in Table 7.

Table 7 association degree for engine lubricants $R$

\begin{tabular}{|c|c|c|}
\hline $\mathrm{K}_{1}(\mathrm{R})$ & $\mathrm{K}_{2}(\mathrm{R})$ & $\mathrm{K}_{3}(\mathrm{R})$ \\
\hline 2.8627 & -0.1894 & -0.6042 \\
\hline
\end{tabular}

(5) Normalization process, as shown in Table 8.

Table 8 normalization process

\begin{tabular}{|l|l|l|}
\hline$\overline{K_{1}}(R)$ & $\overline{K_{2}}(R)$ & $\overline{K_{3}}(R)$ \\
\hline
\end{tabular}

(6) Determine the engine wear levels. According to the definition of wear level, $\bar{J}=1.1069$ is obtained: To test the scientific and effective of this method, Ferrograph of ZTP-X2 is applied to measured the same oil samples, and the characteristic parameters of wear intensity index IS $=218.08$ has been obtained, which is in the region below the baseline, indicating that the wear condition is normal, and the engine can safely and reliably work.

\section{Conclusions}

Through the demonstration above, it shows that there are three advantages to evaluate the engine wear state applying matter-element analysis method. That is, (1) scientific, this method can calculate the associated value, and then get the precise level of engine wear state, which makes the evaluation more accurate and scientific; (2) impartiality, the method reduces the impact of human factors in the fuzzy evaluation method, which makes the evaluation more reasonable and impartial; (3) easy to program, due to the formal representation, programming the evaluation software is becoming possible.

\section{References}

[1] Zhang Lingfang, Guo Jianwei. Fuzzy Comprehensive Evaluation of Wear Status on a Type of Engine Group. Lubrication Engineering, 2007, 10(10), p.120-122

[2] Liu Hongying, Wang Lianshe. NON-SUITABILITY FOR MAXIMUM SUBORDINATE DECREE PRINCIPLE IN ENVIRONMENTAL QUALITY FUZZ COMPREHENSIVE EVALUATION. ARID ENVIRONMENTAL MONITORING, 1995, 9(3), p.141-142

[3] Cai Wen. Matter-element Model and Applying. Science and Technology Literature Press, p.267-275

[4] Zhang Yong. NUMERICAL ANALYSIS AND EXPERIMENTAL INVESTIGATION OF OIL SPECTRUM MEASUREMENT FOR THE PARTS OF THE INTERNAL COMBUSTION ENGINE. Chinese Journal of Mechanical Engineering, 2004, 40(3), p.182-185

[5] YU Feng, YANG Cheng-wu. Application of Material-Units-Analysis Method to the Monitoring of Wearing Conditions in Engines Based on Oil Spectrum. Journal of internal combustion engine, 2006.03. 